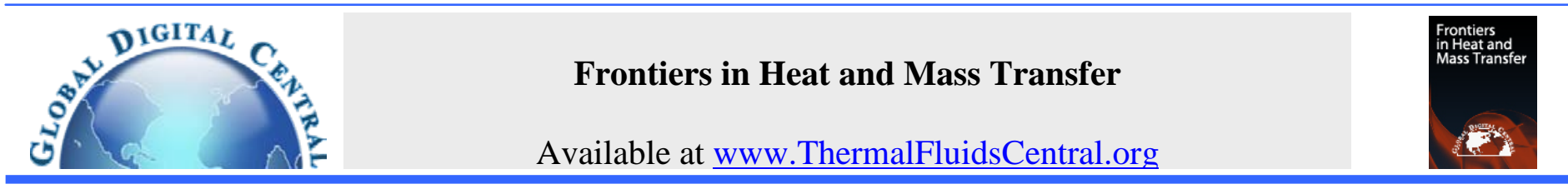

\title{
SIMULATION OF EMBOLIZATION PARTICLE TRAJECTORIES
}

\author{
Nessa Johnson, John Abraham*, Zach Helgeson, Michael Hennessey \\ University of St. Thomas, School of Engineering, St. Paul, MN, 55105-1079, USA
}

\begin{abstract}
A numerical simulation has been performed on the hemodynamics associated with embolization procedures. The flow geometry includes a multibranch artery which is upstream of a targeted tumor. During the procedure, drug-eluting particles are released into the local arterial geometry and are carried downstream by the flowing blood. The intention is to cause embolization of a daughter artery which feeds the tumor. As particles are injected into the blood stream, and as the embolization progresses, it is possible for the particulates to substantially alter the blood flow in the main artery. This alteration may lead to a maldistribution of blood flow and/or a diversion of the particulates from their intended target. The diversion of drug-eluting particulates may become severe as the particles accumulate near the targeted tumor. A consequence of the alteration of flow patterns is that diverted particulates may be sent to untargeted tissue which is otherwise healthy. The simulations completed here were verified with benchtop fluid-flow experiments. The results of the study suggest that late in the embolization procedure, a significant diversion of particulates occurs. Also, it was discovered that the degree of diversion was sensitive to the injection location in the upstream artery and the particle size but not to the injection velocity.
\end{abstract}

Keywords: embolization, computational fluid dynamics, drug-eluting particles, hemodynamics

\section{INTRODUCTION}

Embolization is a medical treatment which involves the purposeful creation of emboli within the arterial system. The intention is to block part of the artery system for medical advantage. One situation in which embolization procedures are performed include the treatment of aneurysms. There, a region of the blood vessel is weakened and can be stretched into a large balloon-shape. An embolization procedure can be used to block the entrance of the aneurysms to isolate the weakened region from the higher pressures which characterize the main blood stream.

Another case in which embolizations are used is in the treatment of uterine fibroids. In these cases, an unwanted, non-cancerous tumor grows on the lining of the uterus. Common treatments include hysterectomy, embolization, or endometrial ablation. For the case of embolization, the arteries which feed the tumor are located and blocked. The result of the blockage is that the tumor is starved of life-giving nutrients which were, until the embolization procedure, supplied by the blood.

Other applications of embolization treatments are obstructive uropathy, hydronephrotic kidneys, and cancer. For cases with cancer, embolization procedures are used to block arteries which feed cancerous tumors. Similar to situations with uterine fibroids, when cancerous tumors are targeted, the goal of the treatment is to occlude blood vessels that supply tumors with nutrition.

The actual occlusion can be achieved by a number of devices. Some devices are coils which block the arterial passage. Other variants are plugs or gelfoam agents. Another occlusion method is through the use of injected microspheres. Often, these micro-spheres are created as drug-eluting particulates so that their injection serves a two-fold purpose. First, the particles block blood flow to the targeted region. Second, the particles slowly release anti-cancer medication. This double-form of attack is thought to increase the success of embolization treatments of cancer.

The procedure of embolization with drug-eluting particles requires the involvement of an interventional radiologist and the procedures are typically carried out in a controlled clinical setting. During the procedure, flow is often measured so that the radiologist can assess the degree of occlusion. The surgeon injects a slurry with particles that are in suspension.

The method of injection involves a great deal of surgical skill and the operating parameters vary from surgeon to surgeon. Among the parameters are: the speed of the injection, the location of the injection port within the artery cross section, the duration of the injection, the total injected volume, the injection angle, the size of the particulates, and the slurry composition. While it is expected that these parameters will affect the clinical outcome, it is not known how this effect occurs. To the best knowledge of the authors, there is little discussion in the literature about the relationship between operating parameters and success.

Summary studies on embolization have been presented in (Golzarian, 2009, Laurent, 2007, and Johnson et al. 2010). Those studies focused on the clinical applications and benefits from the procedure. Another study, (Basciano, 2010), considered the trajectory of the particulates within the arterial system. They focused particular attention on the nature of curved geometries and its impact on the final destination of the particles. This study also determined how the variations in flow due to the pulsating cardiac cycle affected the embolization procedure.

A brief overview of the present understanding of embolization procedures suggests that much of the control of the operating parameters is based on the discretion of the surgeon. Also, control over those parameters is very crude. In many cases, treatment is continued until a visual observation of decreased blood flow is observed. To our best knowledge, there are no detailed assessments of the influence of parameters on the procedure quality. In particular, we have not found

*Corresponding author email: jpabraham@stthomas.edu 
any studies which discuss the interaction of blood flow with particulates and the potential that the presence of particulates might impact the distribution of flow in the arterial system.

Of particular interest is the potential that the occlusion particles might cause a redirection of blood flow to different daughter artery paths. Also, as the procedure progresses, it is possible that the occlusion might cause its own redirection so that subsequent particles do not travel to the targeted location. This potential effect might limit the cumulative effect of particles as the procedure continues. In fact, if drug-eluting particles are redirected to otherwise healthy tissue, it is possible that the anti-cancer agents may cause harm to the untargeted tissue.

To investigate these interactions, a three-dimensional unsteady numerical simulation was completed which included the blood flow in the arterial system and the suspended particles as well. Our study evaluates the impacts of a number of operational settings, including (1) the extent of downstream blockage, (2) the injection velocity of the particles, (3) the location of the injection port, and (4) the particle sizes. The primary goal of the study is to determine which parameters are most influential on determining the trajectory of the particulates and the locations at which they accumulate. It is hoped that with the information obtained in this study, it will be possible to provide guidance to surgeons during future procedures.

\section{NUMERICAL MODEL}

An illustration of a generic arterial geometry is shown in Fig. 1. Included there is a single inlet with three downstream daughter branches. At the termination of one branch, a tumor is depicted. This tumor represents the targeted tissue for the procedure. The injections will be made upstream of all the daughter branches. A major goal of the study is to ensure that the injected particles reach the targeted tumor without being diverted down a branching artery.

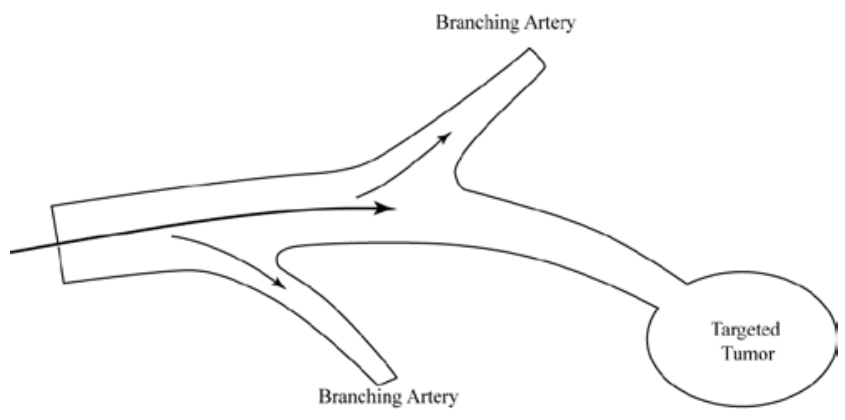

Fig. 1 Artery geometry feeding a targeted tumor (Johnson et al., 2010).

Embolization particulates can be large in size (compared to the artery diameter) and their presence may affect the flow field in their neighborhood. This is particularly likely if the particles are traveling at a velocity that differs from that of the nearby bloodstream. Figure 2 has been prepared to illustrate just this occurrence. There, a series of images shows the progression of a particle down an artery. Arrows are used to represent the blood flow. Darker, heavy arrows signify faster flow, whereas lighter arrows show slower flow. As the particle passes downstream and moves past branch inlets, it reduces the flow that can travel into the inlet and thereby increases the flow elsewhere.

A second issue to be dealt with is whether a downstream occlusion can cause similar redistributions of flow upstream of the targeted site. To illustrate this issue, Fig. 3 has been prepared. It shows a series of three images with progressively more occlusion. By the third image, occluding particles fill the artery which feeds the targeted tumor. The collection of particles increases the resistance to fluid flow at that location, and causes flow bypass upstream.
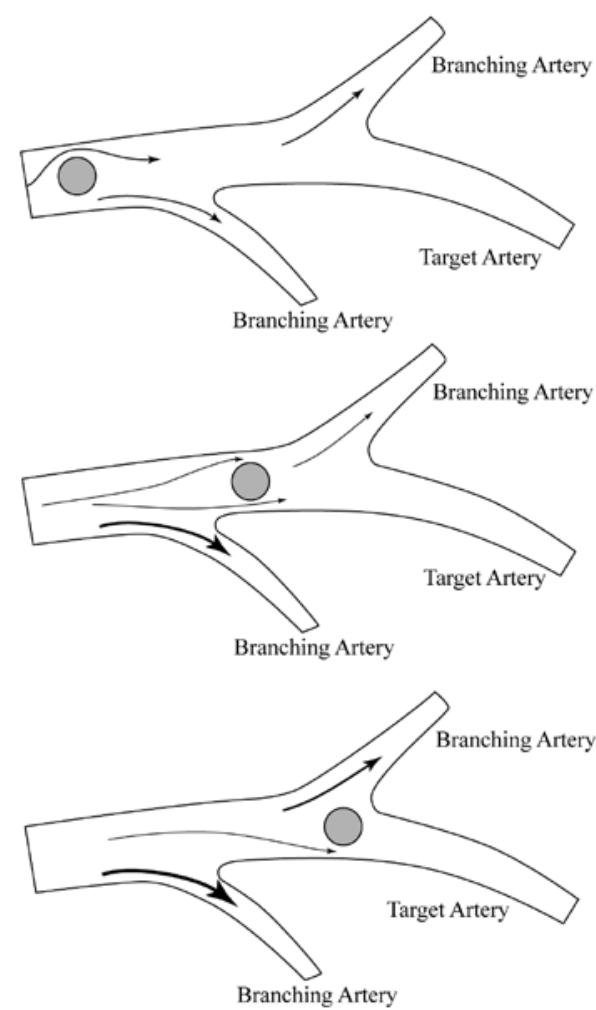

Fig. 2 Flow patterns at sequential instants (Johnson et al., 2010).

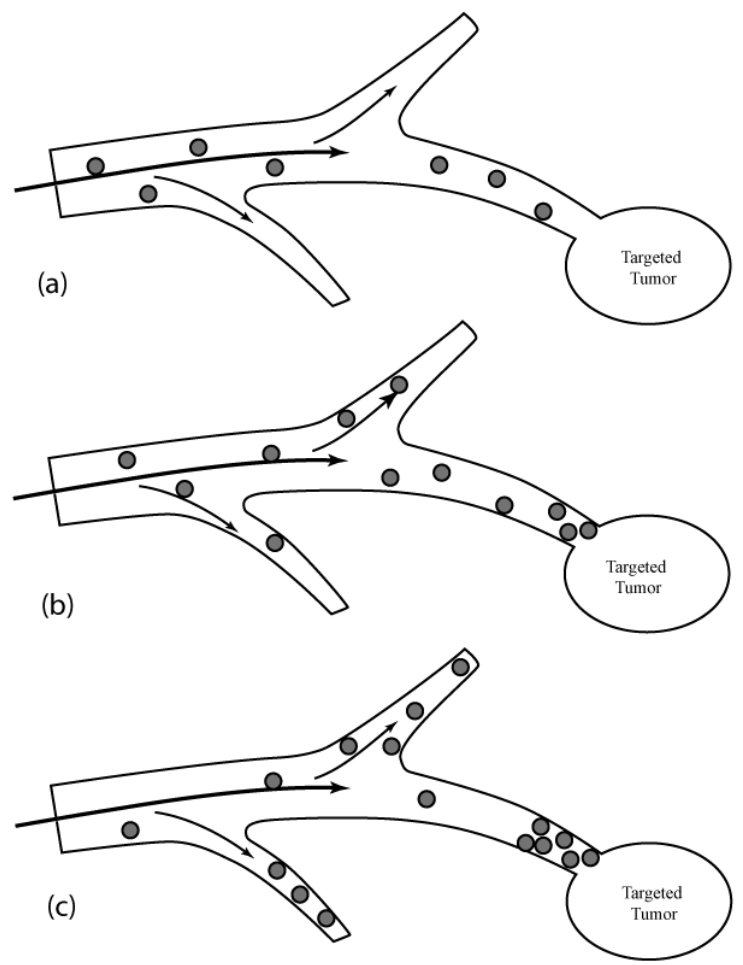

Fig. 3 Alterations in particle trajectories during an embolization procedure (Johnson et al., 2010).

The physiologic dimensions used in the simulations were taken from the typical anatomy of the superficial-femoral region. A computer generated image using in the simulations is shown in Fig. 4. There, a 
single inlet is noted along with three outlets. The diameter of the inlet is $4.95 \mathrm{~mm}$. The outlet diameters are, in order from left to right, 3.12, 3.07 , and $3.70 \mathrm{~mm}$. This geometry was replicated with glass tubing so that benchtop tests could be made which would validate the simulation work.

In the present investigation, the artery geometry and the flowrates guaranteed that the flow would be laminar. The simulations were carried out with an unsteady solution of the governing equations of flow, Newtonian fluid properties were used (which matched the benchtop experiments).

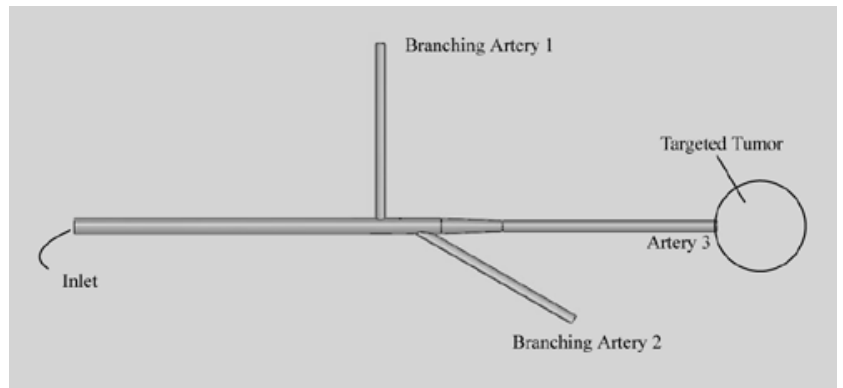

Fig. 4 An artery segment and a targeted tumor (Johnson et al., 2010).

The model requires the simultaneous solution of a number of coupled, partial differential equations. First amongst these is the continuity equation for conservation of mass and the three equations of momentum conservation for the three coordinate directions. These equations are expressed in tensor form as

$\frac{\partial u_{i}}{\partial x_{i}}=0$

and

$\rho \frac{\partial u_{j}}{\partial t}+\rho\left(u_{i} \frac{\partial u_{j}}{\partial x_{i}}\right)=-\frac{\partial p}{d x_{j}}+\mu\left(\frac{\partial^{2} u_{j}}{\partial x_{i}^{2}}\right)$

A Lagrangian method was used to track the location and velocity of individual particles. The tracking is accomplished by instantaneous momentum balances on each injected particle. The momentum balance relies upon a knowledge of the forces which act on the particle. Those forces include drag, buoyancy, and pressure, as indicated in Eq. (3). The combination of these forces gives rise to a change in the particle velocity, $\overrightarrow{V_{p}}$.

$$
m_{p} \frac{d \vec{V}_{p}}{d t}=\vec{F}_{\text {drag }}+\vec{F}_{\text {buoyancy }}+\vec{F}_{\text {pressure }}
$$

The drag forces are a result of the difference of velocity between the particle and the neighboring fluid. The force is found using a drag coefficient calculated from (Schiller and Naumann, 1935) which is then inserted into Eq. (4) for the drag-force calculation. There, the slip velocity represents the difference in velocity between the particle and the local fluid. This model for calculating drag force is based on a spherical, non-deformable object immersed in a fluid region. This limitation is appropriate for the present situation - the drug-eluting particles are approximately spherical and rigid.

$$
\begin{aligned}
& \vec{F}_{\text {drag }}=\frac{1}{2} C_{D} \rho \vec{V}_{\text {slip }} \cdot\left|\vec{V}_{\text {slip }}\right| \\
& =\frac{1}{2} C_{D} \rho\left(\vec{V}_{f}-\vec{V}_{p}\right) \cdot\left|\vec{V}_{f}-\vec{V}_{p}\right|
\end{aligned}
$$

The next force pertains to buoyancy and is easily calculated as the difference between the weight of the particle and the weight of the displaced fluid. Its expression is given in Eq. (5), and the direction of this force is dictated by the local gravitational acceleration.

$$
\vec{F}_{\text {buoyancy }}=\left(m_{p}-m_{f}\right) \vec{g}
$$

Finally, account must also be made of the force caused by local pressure gradients. It is calculated from Eq. (6).

$$
\vec{F}_{\text {pressure }}=-\frac{m_{f}}{\rho} \nabla p
$$

The calculations were completed using the commercial software CFX version 12.0. This computational fluid dynamic code is part of the ANSYS software suite. CFX uses a finite-volume approach to ensure conservation of mass and momentum is obeyed at all control volumes. The numerical scheme is a false-transient wherein calculations at each time step are marched forward in a fictitious time until convergence is achieved. A fully implicit backward-Euler algorithm is used to march forward in real time. This approach is of first-order accuracy in time.

Coupling of the velocity-pressure equations was achieved on a non-staggered, collocated grid using the techniques developed by Rhie and Chow (1982) and Majumdar (1988). The inclusion of pressuresmoothing terms in the mass conservation equation suppresses oscillations which can occur when both the velocity and pressure are evaluated at coincident nodal locations.

The advection term in the momentum equations was evaluated by using the upwind values of the momentum flux, supplemented with an advection-correction term. The correction term reduces the occurrence of numerical false diffusion and is of second-order accuracy. Details of the advection treatment can be found in Barth and Jesperson (1989).

Highly accurate calculations have been successfully completed using this numerical approach and have been reported in Abraham et al., (2008), Abraham et al., (2008), Abraham et al., (2009), and Sparrow et al., (2008), and Lovik et al., (2009).

The computational mesh which was used to subdivide the domain is shown in Fig. 5. It can be seen there that the global mesh is quite fine, and in particular, the wall elements are extremely refined. This refinement was accomplished in order to capture high-gradients which might occur in the boundary layer region. The image shows a cross section of the mesh, as well as an image of the mesh deployed along a portion of the artery wall. Approximately 6 million elements were utilized to complete the calculations. During the calculations, it was found that this number of elements was sufficient to ensure a meshindependent solution. The determination of mesh independence was based on a detailed comparison of the flow results using two different meshes with 3 million and 6 million elements, respectively. A comparison of the flow rates out the respective downstream branches was made with solutions obtained from these meshes. It was found that the variations in flowrates showed less than $2.3 \%$ difference; this finding justified the conclusion of mesh independence.

The boundary conditions used in the simulation were a uniform velocity at the inlet of $34.6 \mathrm{~mm} / \mathrm{sec}$. This value corresponds to a volumetric flowrate of $40 \mathrm{ml} /$ minute. At the three downstream exits, values of pressure of 0 gauge were used. For all transmitted variables, a zero condition was used on the second derivative. Water was the 
simulated fluid so that the calculations could be compared with the water-based benchtop experiments.

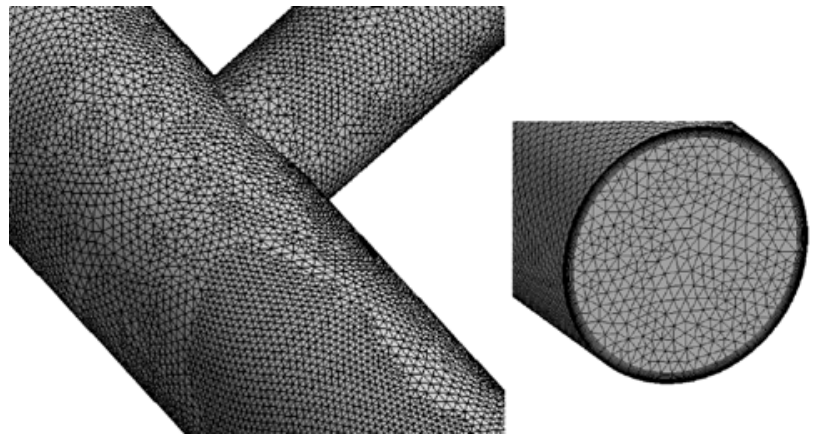

Fig. 5 The computational mesh (Johnson et al., 2010).

\subsection{Validation of the Simulations}

As mentioned in the foregoing section, comparisons between benchtop tests with glass-tube arteries and numerical simulations were performed to validate the simulation procedure. The comparison was made on the outflow extracted from each of the three downstream outlets. Two measures of outflow were made. In the first, the actual liquid flowrate was measured. In the second, the rate of particulate flowrate was determined. During the experiments, a particle-laden slurry was used. Flowrate was captured at each outlet and the solid and liquid phases were separated and measured independently. The information shown in Figs. 6 and 7 show, respectively, the liquid and particulate flowrate comparisons between the experiments and the simulations. The close agreement between these results provided added confidence in the simulations.

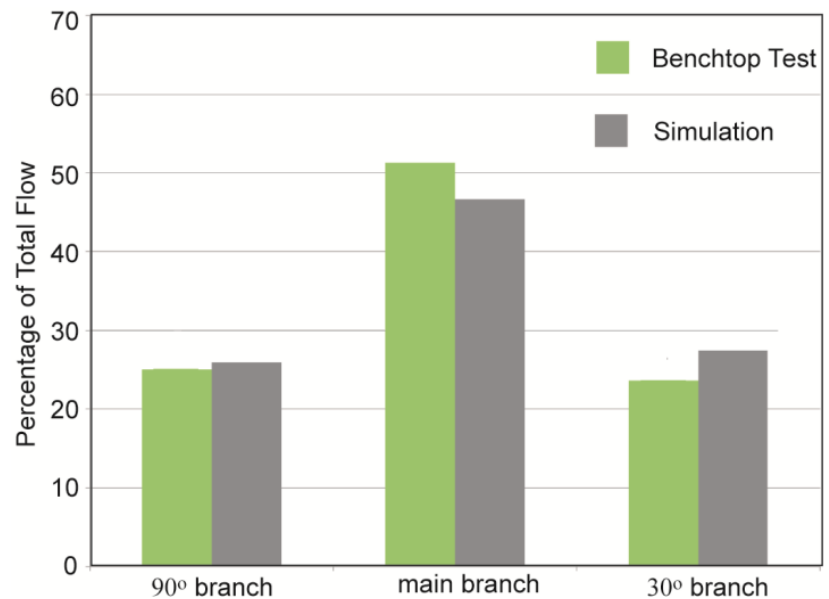

Fig. 6 Comparison of benchtop test with numerical simulations of fluid flowrates at exits of the glass model shown in Fig. 4 (Johnson et al., 2010).

Following the validation of the simulations through the experiments, it was possible to begin the investigation of trajectories of embolizing particles. The first stage of the investigation was the simulation of a wide range of particle sizes to determine the impact of particle size on trajectory. In this stage, particles in the range $2.5 \%<$ $d_{\text {sphere }} / d_{\text {artery }}<20 \%$ were released at the center of the inlet. This range of diameter ratios corresponds to sphere diameters that varied from $125-1000 \mu \mathrm{m}$. In all cases, the initial axial velocity of the particles was $25 \%$ that of the inlet blood velocity $\left(V_{\text {particle }}=8.65 \mathrm{~mm} / \mathrm{s}\right.$ ).

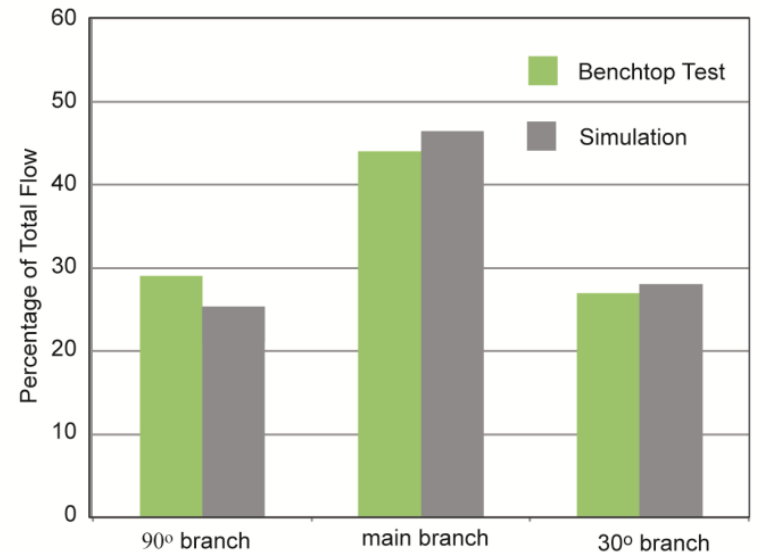

Fig. 7 Comparison of benchtop test with numerical simulations of particulate flowrates at exits of the glass model shown in Fig. 4 (Johnson et al., 2010).

As the simulated particle traveled through the artery, careful attention was paid to the instantaneous flowrates at each exit port. At all instances in the simulation, mass flowrates were calculated so that the timewise variation of flowrate through the individual branches could be found. These calculations formed the basis of the "no blockage" case - a situation where there is no downstream embolism.

Following these calculations, a facsimilar blockage was placed at the outlet of the artery which feeds the targeted tumor. The blockage was in the form of a circular planar wall in the cross section plane. The extent of the blockage was varied parametrically so that its diameter ranged from 2.5 to $3.4 \mathrm{~mm}$. This range corresponds to an area blockage of $46 \%$ to $84 \%$.

In the simulations that followed, two locations were used to introduce the particles into the blood stream. Those two injection locations are noted in Fig. 8 as Injection Location 1 and Injection Location 2. Two locations were used to reflect the near certainty that during an actual procedure, the particulates would not be introduced in the center of the main artery.

A full listing of the investigated cases is shown in Table 1. The table includes information on the size of the injected particles and the extent of embolization blockage. For each case in Table 1, the particles were given an initial velocity which was $25 \%$ that of the blood velocity at the injection location. The density of the material which consitutes the particles is $1600 \mathrm{~kg} / \mathrm{m}^{3}$. This density value is typical of materials which are used to construct the drug-eluting particles.

Table 1 Summary of simulation cases (Johnson et al., 2010).

\begin{tabular}{|l|l|l|}
\hline $\begin{array}{l}\text { Case } \\
\text { No. }\end{array}$ & $\begin{array}{l}\text { Particle Size } \\
(\boldsymbol{\mu m})\end{array}$ & $\begin{array}{l}\mathbf{D}_{\text {blockage }} \mathbf{D}_{\text {outlet }} \\
(\%)\end{array}$ \\
\hline 1 & 125 & 0 \\
\hline 2 & 125 & $68 \%$ \\
\hline 3 & 125 & $81 \%$ \\
\hline 4 & 125 & $92 \%$ \\
\hline 5 & 250 & 0 \\
\hline 6 & 250 & $68 \%$ \\
\hline 7 & 250 & $81 \%$ \\
\hline 8 & 250 & $92 \%$ \\
\hline 9 & 500 & 0 \\
\hline 10 & 500 & $68 \%$ \\
\hline 11 & 500 & $81 \%$ \\
\hline 12 & 500 & $92 \%$ \\
\hline 13 & 1000 & 0 \\
\hline 14 & 1000 & $68 \%$ \\
\hline 15 & 1000 & $81 \%$ \\
\hline 16 & 1000 & $92 \%$ \\
\hline & & \\
\hline
\end{tabular}




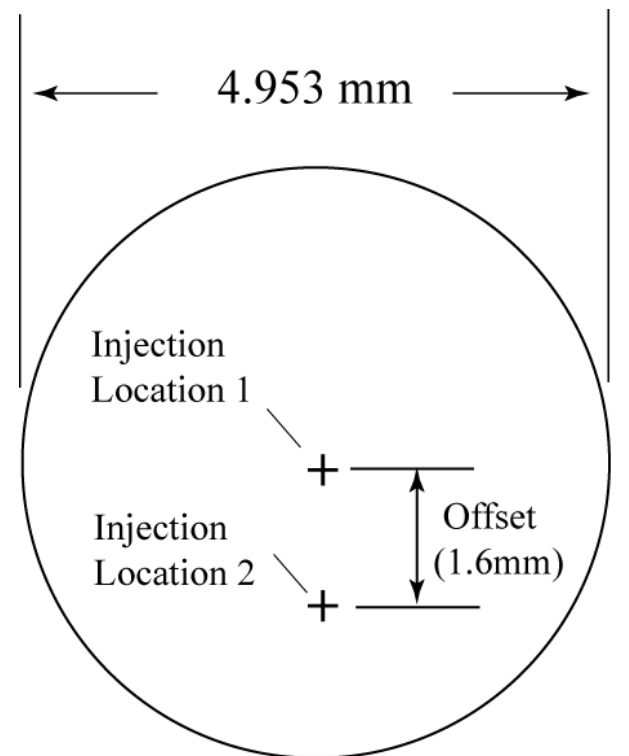

Fig. 8 Inlet cross section showing two injection locations (Johnson et al., 2010).

\section{RESULTS AND DISCUSSION}

The first result to be considered is the potential misdirection of particles down unintended daughter arteries. In the first presentation, a $125 \mu \mathrm{m}$ particle has been released from the center of the inlet plane (Injection Location 1). Initially, there is no downstream blockage in the target artery. The resulting trajectory of the particle is shown graphically in Fig. 9. There, it is seen that the path of the particle is diverted upwards as it passes the first branching artery. Next, the particle is brought toward the center of the channel as it passes the second branching artery. It is seen that despite these diversions, the particle successfully makes its way to the targeted artery. The case shown here, in Fig. 9, corresponds to $\quad$ Case 1 in Table 1.

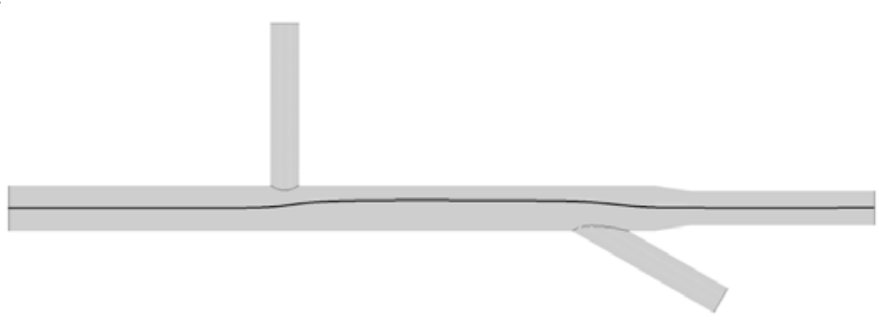

Fig. 9 Trajectory of $125 \mu$ m particle released from Injection Location 1, no downstream blockage (Johnson et al., 2010).

Next, a similar set of results is presented in Fig. 10. Here, however, there is a significant downstream blockage in the target artery. The path shown in Fig. 10 corresponds to Case 4 in Table 1. It is seen that while the particle trajectory deviates more than the path shown in Fig. 9 , the particle's final destination is still down the targeted artery. It can be concluded from this set of results that, for small particles $(125 \mu \mathrm{m})$ and a central injection (Injection Location 1), the presence of a blockage did not prohibit the particle from reaching its target destination.

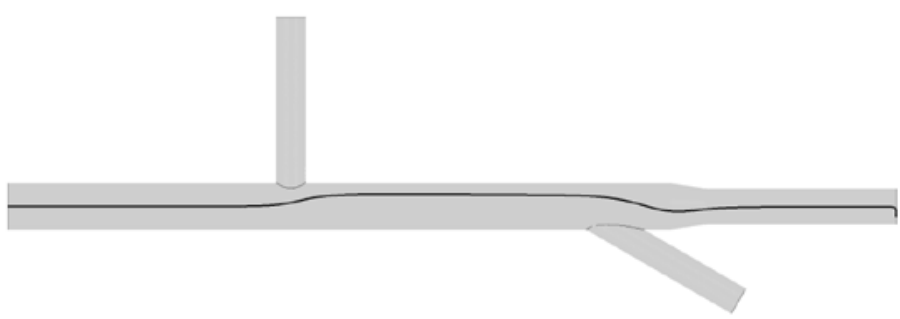

Fig. 10 Trajectory of $125 \mu$ marticle released from Injection Location 1, significant downstream blockage (Johnson et al., 2010).

Results for the remaining cases of Table 1 showed that for larger particles, the particles were also successful in passing into the target artery. Only results for Cases 1 and 4 are displayed for brevity. It is expected that the smaller particles would be more susceptible to diversion than would large particles. Since the smallest particles were not diverted to unintended branches, it was expected that none of the cases in Table 1 would result in diversion. This expectation was realized when the entire set of simulations was completed. It is then concluded that particles of all sizes are able to reach the target region, provided their injection location is in the center of the inlet artery. To reinforce the findings, it was also seen that fluid streamlines released from Injection Location 1 traveled to the targeted artery, regardless of the extent of the downstream blockage. Streamlines are infinitely small fluid particles so they represent a limit of extremely small drug-eluting particles.

The next results to be conveyed show the impact of a non-central injection location (Injection Location 2). These results are shown in Figs. 11 and 12. In Fig. 11, a particle path is displayed for the settings of Case 1 (from Table 1). For Fig. 12, the pathline corresponds to Case 4 from Table 1. It can be seen that when there is no downstream blockage (Fig. 11), the particles that are released from Injection Location 2 are able to pass downstream, into the targeted artery. On the other hand, when there is a significant blockage in the target artery (resulting for a progression of occlusion), the particle is diverted into one of the branching arteries and therefore, it's ultimate destination is to a region of healthy tissue. The full set of results showed that with a lesser amount of downstream blockage (Cases 2 and 3 from Table 1) the particulates were diverted to unintended arteries.

For slight larger particulates $(250 \mu \mathrm{m})$ which are released from Injection Location 2, it was seen that they were diverted down unintended arteries only for the most extreme case of blockage. They are, as expected, less sensitive to redirection as a result of occlusion. For the two largest-sized particles, (500 and $1000 \mu \mathrm{m})$, in no cases were they misdirected to unintended branches.

While these results may have been predicted beforehand, it is clear that injection location is important in determining the ultimate particle destination. Particles which are released more centrally in the facsimile arterial geometry of this investigation are more likely to pass unimpeded to downstream, targeted arteries. On the other hand, smaller particles are more susceptible to misdirection than are larger particles. This finding acknowledges the momentum that dense and large particles have.

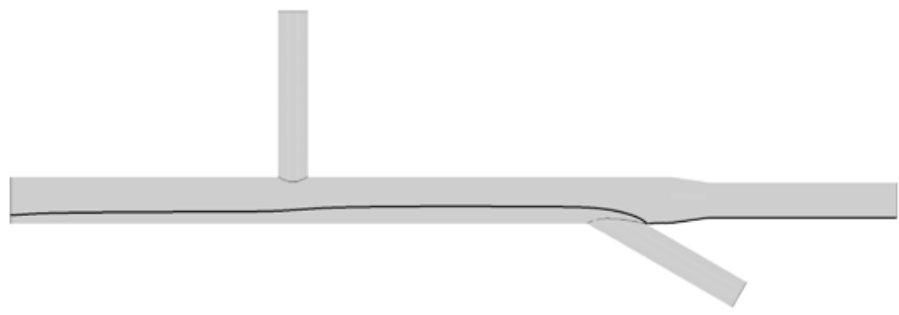

Fig. 11 Trajectory of $125 \mu$ m particle released from Injection Location 2, no downstream blockage (Johnson et al., 2010). 


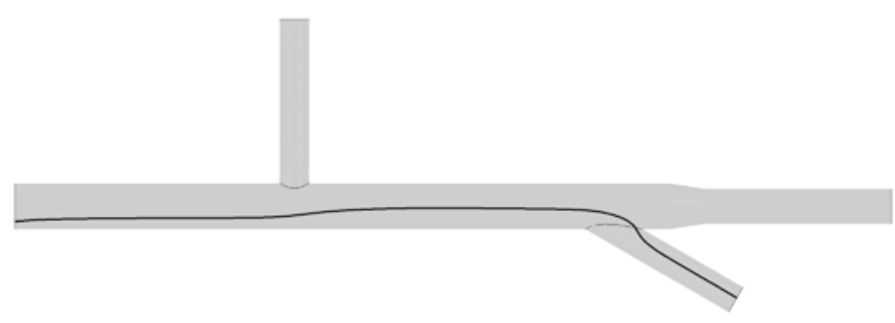

Fig. 12 Trajectory of $125 \mu$ m particle released from Injection Location 2, significant downstream blockage (Johnson et al., 2010).

It is possible to explore the impact of particle size on the potential for misdirection in greater detail by comparing a particle pathline with a co-located streamline. A streamline is the path of an infinitely small parcel of liquid. The potential for redirection is inversely related to the density of the particle in question. More dense particles are less likely to be diverted than are lighter particles or streamlines. In this effort, Fig. 13 has been prepared which shows an image of a streamline (white) and a superimposed particle track (black), both of which originate at Injection Location 2. The relevant case is 16 from Table 1 (a large particle with an extensive blockage). It is seen that the streamline is diverted through the $30^{\circ}$ branch artery while the particle bypasses the branch and travels into the target artery.

In contrast, a similar blockage with a small particle (Case 4 from Table 1) can be seen in Fig. 14. There, both the streamline and the particle travel down the $30^{\circ}$ branch. Again, these results correspond to streamlines and particles which originate from Injection Location 2. A contrast of Figs. 13 and 14 reveal the expected result that larger particles are less likely to be diverted to unintended branches than are small particles. It should be reinforced that the conclusions drawn from this study are related to the simulated arterial geometry shown in Fig. 4. Caution must be exercised before these results are applied to arteries which differ significantly from that shown in the figure.

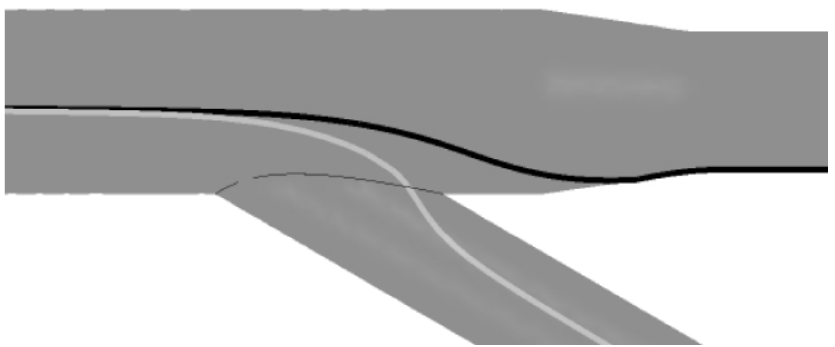

Fig. 13 Image of superimposed streamline (white) and a $1000 \mu \mathrm{m}$ particle track (black) showing diverging paths with a blockage diameter equal to $92 \%$ of the target artery diameter (Johnson et al., 2010).

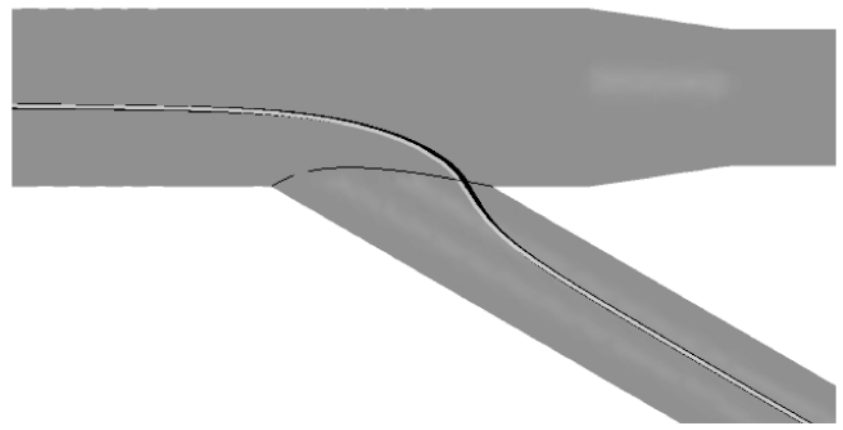

Fig. 14 Image of superimposed streamline (white) and a $125 \mu \mathrm{m}$ particle track (black) showing diverging paths with a blockage diameter equal to 92\% of the target artery diameter (Johnson et al., 2010).
Another important aspect of the present investigation is the determination of whether the presence of particles changes the distribution of liquid which flows through the respective downstream ports. For this study, calculations of the timewise variation of mass flow were required. Those calculations are shown in Eq. (7). The continuous integral for mass flowrate has been replaced by a summation of mass flow through the individual elements which constitute the outlet planes. The numerical integration of Eq. (7) was completed for all 16 cases of Table 1 . For each case, the initial particle velocity was given values of $25 \%, 50 \%$, and $75 \%$ of the local blood flow. Corresponding values of the initial particle velocities are 8.65 $\mathrm{mm} / \mathrm{sec}, 17.3 \mathrm{~mm} / \mathrm{sec}$ and $26.0 \mathrm{~mm} / \mathrm{sec}$.

$$
\dot{m}=\int_{\text {area }} \rho \vec{u} \cdot d \vec{A}=\sum_{i=1}^{n} \rho \vec{u} \cdot \Delta \vec{A}
$$

The aforementioned calculations show that the particles quickly accelerate to the velocity of the local blood flow. The quickness of this acceleration is quantified by the distance over which the acceleration occurred. To illustrate this distance, Fig. 15 has been prepared. That figure illustrates a distance over which a particle achieves a velocity that is $95 \%$ that of the local fluid. The distance is termed the acceleration length.

When the results of the simulations were carefully analyzed, it was seen that for all cases, the acceleration lengths were very short (less than 10 particle diameters in all cases). Values of acceleration lengths are provided for the cases in which the injection velocity of the particles were $25 \%$ that of the local blood velocity are used as representative of the larger set of results.

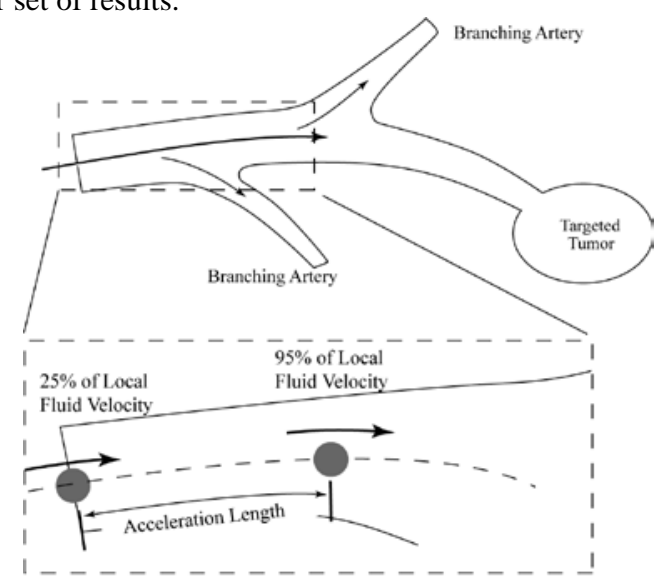

Fig. 15 Schematic showing an accelerating embolizing particle (Johnson et al., 2010).

Table 2 Acceleration lengths for a range of particles with an injected velocity of $8.65 \mathrm{~mm} / \mathrm{s}$ (Johnson et al., 2010).

\begin{tabular}{|l|l|l|}
\hline $\begin{array}{l}\text { Particle Size } \\
(\mu \mathbf{m})\end{array}$ & $\begin{array}{l}\text { Acceleration } \\
\text { Length/D }\end{array}$ & $\begin{array}{l}\text { Acceleration } \\
\text { Length }(\mathbf{m m})\end{array}$ \\
\hline 125 & 0.74 & 0.093 \\
\hline 250 & 2.5 & 0.63 \\
\hline 375 & 4.2 & 1.6 \\
\hline 500 & 5.1 & 3.0 \\
\hline 1000 & 5.9 & 9.8 \\
\hline
\end{tabular}

For cases in which the injection velocities of the particles was closer to that of the local blood flow, the acceleration lengths were even shorter than those shown in Table 2 . These results clearly indicate that the impact of injection velocity on the ultimate destination of the particles is minor and is overwhelmed by the impact of particle size and injection location. This conclusion is reinforced by the fact that 
variations in initial velocity did not impact the ultimate destination of the particles.

The final results which are to be presented correspond to the timewise variation of mass flowrates through each of the artery exits. It was first found that the presence of a particle traveling through the arterial system had virtually no impact on the distribution of fluid flow. This finding is in accord with the knowledge that the particles move with the velocity of the local fluid - they do not disturb the flow field in their near vicinity (the variation in mass flowrate was less than $1 \%$ as particles traveled through the system). On the other hand, the progression of occlusion in the target artery has the potential of causing a flow redistribution. Information was obtained on the apportionment of flow through each of the daughter arteries with various amounts of occlusion. The results are presented in Table 3 . The arteries are numbered right-to-left in Fig. 4 . Artery 1 has a $90^{\circ}$ branch, Artery 2 branches at $30^{\circ}$, and Artery 3 is the main branch.

It can be seen that for no blockage, a large portion of the flow passes through the target artery (Artery 3). As the extent of blockage increases, the amount of flow through Artery 3 decreases while the flow through Arteries 1 and 2 increase.

Table 3 Effect of downstream blockage on artery-branch flowrates (Johnson et al., 2010).

\begin{tabular}{|l|l|l|l|}
\hline $\mathbf{D}_{\text {blockage }} / \mathbf{D}_{\text {outlet }} \mathbf{( \% )}$ & Artery 1 & Artery 2 & Artery 3 \\
\hline 0 & $26.0 \%$ & $28.3 \%$ & $45.7 \%$ \\
\hline $68 \%$ & $31.3 \%$ & $33.0 \%$ & $35.7 \%$ \\
\hline $81 \%$ & $34.8 \%$ & $37.2 \%$ & $28.0 \%$ \\
\hline $92 \%$ & $39.6 \%$ & $42.5 \%$ & $17.9 \%$ \\
\hline
\end{tabular}

\section{CONCLUDING REMARKS}

This study has assessed the impacts of various embolization parameters on the flow distribution and the particle trajectories which are used to create the embolization. The parameters which were considered include: (a) the injection location, (b) the particle size, (c) the initial velocity of the particles, and (d) the presence or absence of a downstream blockage. The impacts of these parameters on the potential for redirecting particles to untargeted arteries and on the distribution of flow through each of three downstream branch arteries was determined.

It was found that the extent of downstream blockage had a major impact on both the particle trajectory and on the distribution of fluid flow. As the extent of blockage was increased, particles were more likely to be sent down untargeted branches to otherwise healthy tissue. Similarly, as blockage increases, flow is redistributed to other branches.

It was also found that there is a notable impact of the particle size on the trajectories of the drug-eluting spheres. Specifically, larger particles are more likely to travel unimpeded downstream to the targeted artery, even when there is an appreciable downstream blockage.

Recognizing that control of the injection location during surgery would be inexact, two injection locations were considered in this study. The first injection location was centrally positioned at the artery inlet. The second location was off-center in the same plane. It was found that particles released from a non-central location are more susceptible to being redirected to untargeted branches.

Finally, the effect of injection velocity was found to be negligible. Particles released at the inlet quickly accelerate to the local velocity of the blood so that the memory of the injection velocity is quickly lost.
It is hoped that these findings might provide guidance to surgeons who currently use embolization therapy in the treatment of various medical afflictions, including cancer.

\section{REFERENCES}

Abraham, J.P., Sparrow, E.M., and Lovik, R.D., 2008, "Unsteady, Three-Dimensional Fluid Mechanics of Blood Flow in PlaqueNarrowed and Plaque-Free Arteries", International Journal of Heat and Mass Transfer, 51, 5633-5641.

doi: 10.1016/j.ijheatmasstransfer.2008.04.038

Abraham, J.P., Sparrow, E.M., and Tong, J.C.K., 2009 , “Heat Transfer in All Pipe-Flow Regimes - Laminar, Transitional/Intermittent, and Turbulent”, International Journal of Heat and Mass Transfer, 52, 557563.

doi: 10.1016/j.ijheatmasstransfer.2008.07.009

Johnson, N.N., Abraham, J. P., Z.I. Helgeson, and M.P. Hennessey, "Numerical Simulation of Blood Flow in the Presence of Embolizing Agents”, ASME International Congress and Expo, Vancouver, CA, November 12-18, 2010.

Lovik, R.D., Abraham, J.P., Minkowycz, W.J., and Sparrow, E.M., 2009, "Laminarization and Turbulentization in a Pulsatile Pipe Flow", Numerical Heat Transfer A, 56, 861-879, 2009.

doi: 10.1080/10407780903466568

Golzarian, J., 2009, “An Overview of Embolics”, Endovascular Today, 8, 37-67.

Laurent, A., 2007, "Microspheres and Nonspherical Particles for Embolization”, Techniques in Vascular and Interventional Radiology, 10, 248-256.

doi:10.1053/j.tvir.2008.03.010

Basciano, C.A., Kleinstreuer, C., Kennedy, A.S., Dezarin, W.A., and Childress, E., 2010, "Computer Modeling of Controlled Microsphere Release and Targeting in a Representative Hepatic Artery System", Annals of Biomedical Engineering, 38, 1862-1879.

doi: 10.1007/s10439-010-9955-z

Schiller, L. and Naumann, Z., 1935, “A Drag Coefficient Correlation”, Z. Ver. Deutsch. Ing., 77, 318-324.

Sparrow, E.M., Tong, J.C.K., and Abraham, J.P., 2008, "Fluid Flow in a System with Separate Laminar and Turbulent Zones", Numerical Heat Transfer A, 53, 341-353, 2008.

doi: $10.1080 / 10407780701454162$

Rhie, W.C., and Chow, W.L., 1983, "A Numerical Study of the Turbulent Flow Past an Isolated Airfoil with Trailing Edge Separation", AIAA Paper no. 82-0998.

Majumdar, S., 1988, "Role of Underrelaxation in Momentum Interpolation for Calculation of Flow with Nonstaggered Grids", Numerical Heat Transfer, 13, 125-132.

doi: $10.1080 / 10407788808913607$

Barth, T.J., and Jesperson, D.C., 1989, “The Design and Applications of Upwind Schemes on Unstructured Meshes”, AIAA Paper no. 89-0366. 